\title{
Relationships between electrical conductivity and total and sulphated ash contents in Basque honeys
}

\author{
MT Sancho, S Muniategui, MP Sánchez, JF Huidobro*, J Simal \\ Facultad de Farmacia, Departamento de Química Analitica, Nutrición y Bromatología, \\ Area de Nutrición y Bromatología, 15706 Santiago de Compostela, La Coruña, Spain
}

(Received 9 November 1989; accepted 30 May 1991)

\begin{abstract}
Summary - In 30 honeys of the Basque country ( $N$ spain), linear relationships have been found between electrical conductivity, measured at $20^{\circ} \mathrm{C}$ in solutions containing $10 \mathrm{~g}$ of honey dissolved in $75 \mathrm{ml}$ of water, and total and sulphated ash contents:

total ash $(\%)=0.083$ electrical conductivity -0.092

$(r=0.964, P$ value $=96.3 \%)$;

sulphated ash $(\%)=0.121$ electrical conductivity -0.097

$(r=0.981, P$ value $=99.4 \%)$.

The straightforwardness of electrical conductivity measurements in honey might make these relationships a useful means of calculating ash content.
\end{abstract}

honey / electrical conductivity / ash content / Spain

\section{INTRODUCTION}

In sugar solutions and refined sugars, electrical conductivity has been related to ash content by a number of authors (Zerban and Sattler, 1930; Pearson, 1962). In honey, electrical conductivity is related to organic acid, protein and polyol contents (Crane, 1975). Accorti et al (1986, 1987) found a correlation between electrical conductivity (of $20 \%$ honey solutions based on the honey dry weight) and the total ash content. The ash content in honey varies widely (White, 1978). Under "the essential composition and quality factors for honey" the Codex Alimentarius Commission (1969) specifies: "Mineral content (ash): not more than $0.6 \%$. In honeydew honey and blends of honeydew and blossom honey: not more than $1.0 \%$.

In the literature there are no data on the mineral content of honey expressed as sulphated ash. The determination of the sulphated ash content (as for sugar and sugar products) would lead to less loss of volatile components (which in sulphated form are not readily volatile), and to less uncertainty in measurements,

\footnotetext{
* Correspondence and reprints
} 
since the absolute quantity of ash is greater.

It is time-consuming and laborious to directly determine the ash content of honey. The work presented here demonstrates a relationship between electrical conductivity, which is quickly and easily determined at $20^{\circ} \mathrm{C}$, and ash content, on Basque honeys.

\section{MATERIALS AND METHODS}

\section{Samples}

The study involved 30 Basque honeys harvested in autumn 1987 originating from the chief honey production areas. They were collected representatively from mountain, coastal and plain areas. Microscopic analysis of their sediments revealed that 29 samples were from nectar:

- 15 samples were polyfloral honeys Nos 5,6 , $7,10,11,12,13,14,16,18,19,20,25,26$ and 29;

- 6 samples were Ericaceae honeys Nos 1, 21, 22, 24, 27 and 28;

- 4 samples were Eucalyptus sp honeys Nos 2, 4, 8 and 9;

- 2 samples were Castanea sativa Miller honeys Nos 3 and 17;

- 1 sample was Rubus sp honey No 15;

- 1 sample was Lotus sp honey No 23;

- only 1 sample (No 30) was from nectar and honeydew.

\section{Electrical conductivity}

Measurements were taken with a Radiometer conductimeter with a $1-\mathrm{cm}$ Radiometer electrode. Sample temperature was maintained at $20^{\circ} \mathrm{C}$ using a Haake NBS ultrathermostat and a Jeaner Normalglas thermometer accurate to \pm $0.1^{\circ} \mathrm{C}$. Electrical conductivity was measured in solutions containing $10 \mathrm{~g}$ of honey in $75 \mathrm{ml}$ of water (double-distilled), since Sancho et al (1991) found an excellent correlation $(r=$ 0.9998 ) between the electrical conductivity of these solutions and that measured in honey solutions containing $20 \%$ dry matter (Vorwohl, 1964a, b; Louveaux et al, 1973). The electrical conductivity of honey solution containing $20 \%$ dry matter can be estimated by measuring the electrical conductivity of a solution of $10.00 \mathrm{~g}$ of honey in $75 \mathrm{ml}$ of water and multiplying it by 1.50 (Sancho et al, 1991).

\section{Total ash}

Total ash content was determined by the method of White et al (1962), which is the Official Final Action method for honey of the AOAC (1990). A Tungsram infrared lamp and a Heraeus Hanan oven were used.

\section{Sulphated ash}

Sulphated ash content was determined by the method of Leach and Winton (1920), the Official Final Action method for sugars and sugar products of the AOAC (1990). The infrared lamp described above was used to heat the samples on a hot plate until they were well-carbonized and evaporated after the second addition of sulphuric acid.

The precision of each procedure was determined as follows: electrical conductivity, $\% \mathrm{Cv}=$ 0.86 (Sancho et al, 1991); total ash, \%Cv = 2.02; sulphated ash, $\% \mathrm{Cv}=1.30$.

\section{RESULTS AND DISCUSSION}

Plotting the values (table I) of electrical conductivity at $20{ }^{\circ} \mathrm{C}$ in humid matter against the total (fig 1) and sulphated (fig 2) ash contents of the honeys of the Basque Country gives a linear relation in both cases:

$\%$ total ash $=0.083$ electrical conductivity $-0.092(r=0.964)$; 


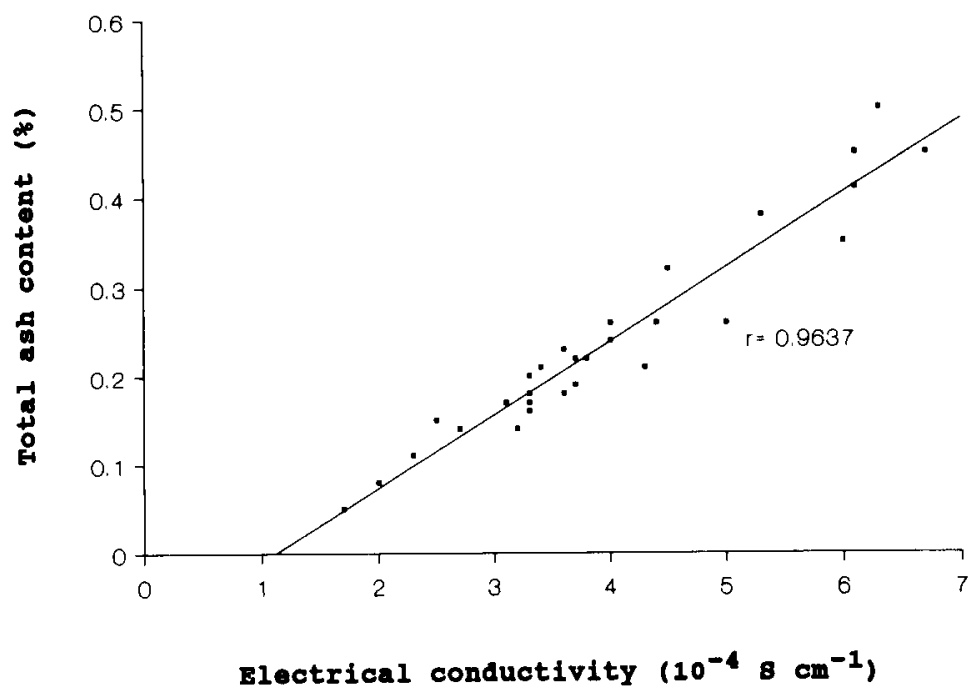

Fig 1. Graph of relation between electrical conductivities $\left(10^{-4} \mathrm{~S} \mathrm{~cm}^{-1}\right)$ of Basque honey solutions in humid matter and their total ash contents $(\%)$.

$\%$ sulphated ash $=0.121$ electrical conductivity $-0.097(r=0.981)$.

Also shown in table I are the theoretical values of the total and sulphated ash contents calculated from these equations. The $t$-test was applied to these data (Biomedical Computers Program PD3, 1985). No significant differences (table II) were indicated between the 2 series of results $(P=$ $96.3 \%$ and $99.4 \%$ for the total and sulphated ash, respectively).

The correlation between electrical conductivity at $20^{\circ} \mathrm{C}$ and total and sulphated ash contents shown in these results indicates the possible influence of mineral components of honey on its electrical conductivity.

The correlation between electrical conductivity and total ash content found in this work is similar to the findings of Accorti et al (1986, 1987). These authors established a relation between the electrical conductivity of $20 \%$ honey solution, based on honey dry weight, and the total ash content given by the equation:

electrical conductivity $=17.1856(\%)$ total ash + 1.4918 where:

$\%$ total ash $=0.058$ electrical conductivity -0.087 .

If the electrical conductivity as determined by Accorti et al $(1986,1987)$ was measured in solutions of $10 \mathrm{~g}$ honey dissolved in $75 \mathrm{ml}$ water, the relationship would be:

$\%$ total ash $=0.087$ electrical conductivity -0.087 .

The similarity between the equation of Accorti et al (1986, 1987) and that of the present study (\% total ash $=0.083$ electri- 
Table l. Results of electrical conductivity $\left(10^{-4} \mathrm{~S} \mathrm{~cm}^{-1}\right)$ measured in humid matter at $20^{\circ} \mathrm{C}$; total and sulphated ash (experimental and calculated) contents (\%P/P) in Basque Country (Spain) honeys.

\begin{tabular}{|c|c|c|c|c|c|}
\hline \multirow[t]{2}{*}{$\begin{array}{c}\text { Sample } \\
\text { No }\end{array}$} & \multirow{2}{*}{$\begin{array}{c}\text { Electrical } \\
\text { conductivity } \\
\left(10^{-4} \mathrm{~S} \mathrm{~cm}^{-1}\right)\end{array}$} & \multicolumn{2}{|c|}{$\begin{array}{c}\text { Total } \\
\text { ash (\%) }\end{array}$} & \multicolumn{2}{|c|}{$\begin{array}{l}\text { Sulphated } \\
\text { ash (\%) }\end{array}$} \\
\hline & & Experimental & Calculated & Experimental & Calculated \\
\hline 1 & 6.3 & 0.50 & 0.43 & 0.74 & 0.67 \\
\hline 2 & 3.8 & 0.22 & 0.22 & 0.40 & 0.36 \\
\hline 3 & 2.7 & 0.14 & 0.13 & 0.24 & 0.23 \\
\hline 4 & 3.7 & 0.19 & 0.21 & 0.35 & 0.35 \\
\hline 5 & 3.1 & 0.17 & 0.16 & 0.28 & 0.28 \\
\hline 6 & 5.0 & 0.26 & 0.32 & 0.46 & 0.51 \\
\hline 7 & 4.3 & 0.21 & 0.26 & 0.38 & 0.42 \\
\hline 8 & 3.7 & 0.19 & 0.21 & 0.31 & 0.35 \\
\hline 9 & 3.3 & 0.20 & 0.18 & 0.32 & 0.30 \\
\hline 10 & 3.6 & 0.23 & 0.21 & 0.34 & 0.34 \\
\hline 11 & 3.3 & 0.18 & 0.18 & 0.31 & 0.30 \\
\hline 12 & 2.3 & 0.11 & 0.10 & 0.18 & 0.18 \\
\hline 13 & 3.3 & 0.17 & 0.18 & 0.29 & 0.30 \\
\hline 14 & 3.2 & 0.14 & 0.17 & 0.27 & 0.29 \\
\hline 15 & 1.7 & 0.05 & 0.05 & 0.14 & 0.11 \\
\hline 16 & 4.0 & 0.24 & 0.24 & 0.38 & 0.39 \\
\hline 17 & 5.3 & 0.38 & 0.35 & 0.54 & 0.55 \\
\hline 18 & 2.5 & 0.15 & 0.11 & 0.22 & 0.21 \\
\hline 19 & 4.0 & 0.26 & 0.24 & 0.35 & 0.39 \\
\hline 20 & 3.3 & 0.16 & 0.18 & 0.25 & 0.30 \\
\hline 21 & 3.4 & 0.21 & 0.19 & 0.33 & 0.32 \\
\hline 22 & 6.1 & 0.45 & 0.41 & 0.67 & 0.64 \\
\hline 23 & 3.6 & 0.18 & 0.21 & 0.32 & 0.34 \\
\hline 24 & 6.7 & 0.45 & 0.46 & 0.66 & 0.72 \\
\hline 25 & 4.5 & 0.32 & 0.28 & 0.47 & 0.45 \\
\hline 26 & 3.7 & 0.22 & 0.21 & 0.37 & 0.35 \\
\hline 27 & 6.0 & 0.35 & 0.40 & 0.65 & 0.63 \\
\hline 28 & 4.4 & 0.26 & 0.27 & 0.49 & 0.44 \\
\hline 29 & 2.0 & 0.08 & 0.07 & 0.16 & 0.15 \\
\hline 30 & 6.1 & 0.41 & 0.41 & 0.65 & 0.64 \\
\hline
\end{tabular}

cal conductivity - 0.092), shows that total ash content of honey can be calculated from the value of the electrical conductivity. The greater quantity of sulphated ash explains the higher correlation for sulphated ash content and the lower \%Cv.

We have shown that, at least for the Basque honeys harvested in autumn
1987, total and sulphated ash contents in honey can be estimated from the value of the electrical conductivity at $20^{\circ} \mathrm{C}$ measured in solutions of $10 \mathrm{~g}$ honey dissolved in $75 \mathrm{ml}$ of water. An extension of this study might be to determine whether the same relationships exist in other honeys of different origins (nectar on honeydew), 
Table II. Analysis of data from methods comparison ( $t$-test using BMDP-P3D statistical program).

Experimental total ash vs calculated total ash contents

\begin{tabular}{|c|c|c|c|c|c|}
\hline & & & Mean & 0.2360 & 0.2347 \\
\hline$t$ & $P$-value & DF & $\begin{array}{l}\text { St dev } \\
\text { SEM }\end{array}$ & $\begin{array}{l}0.1119 \\
0.0204\end{array}$ & $\begin{array}{l}0.1075 \\
0.0196\end{array}$ \\
\hline 0.05 & 0.9626 & 58 & $\begin{array}{l}\text { Sample size } \\
\text { Maximum } \\
\text { Minimum }\end{array}$ & $\begin{array}{l}30 \\
0.5000 \\
0.05\end{array}$ & $\begin{array}{l}30 \\
0.46 \\
0.05\end{array}$ \\
\hline
\end{tabular}

Experimental sulfate ash vs calculated sulphated ash contents

\begin{tabular}{|c|c|c|c|c|c|}
\hline$t$ & $P$-value & DF & $\begin{array}{l}\text { Mean } \\
\text { St dev } \\
\text { SEM }\end{array}$ & $\begin{array}{l}0.3840 \\
0.1610 \\
0.0294\end{array}$ & $\begin{array}{l}0.3837 \\
0.1580 \\
0.0288\end{array}$ \\
\hline 0.01 & 0.9936 & 58 & $\begin{array}{l}\text { Sample size } \\
\text { Maximum } \\
\text { Minimum }\end{array}$ & $\begin{array}{l}30 \\
0.74 \\
0.14\end{array}$ & $\begin{array}{l}30 \\
0.72 \\
0.11\end{array}$ \\
\hline
\end{tabular}

$P$-Value: probability value. DF: degrees of freedom. St dev: standard deviation. SEM: standard error of the mean $(\mathrm{S} / \sqrt{\mathrm{N}})$.

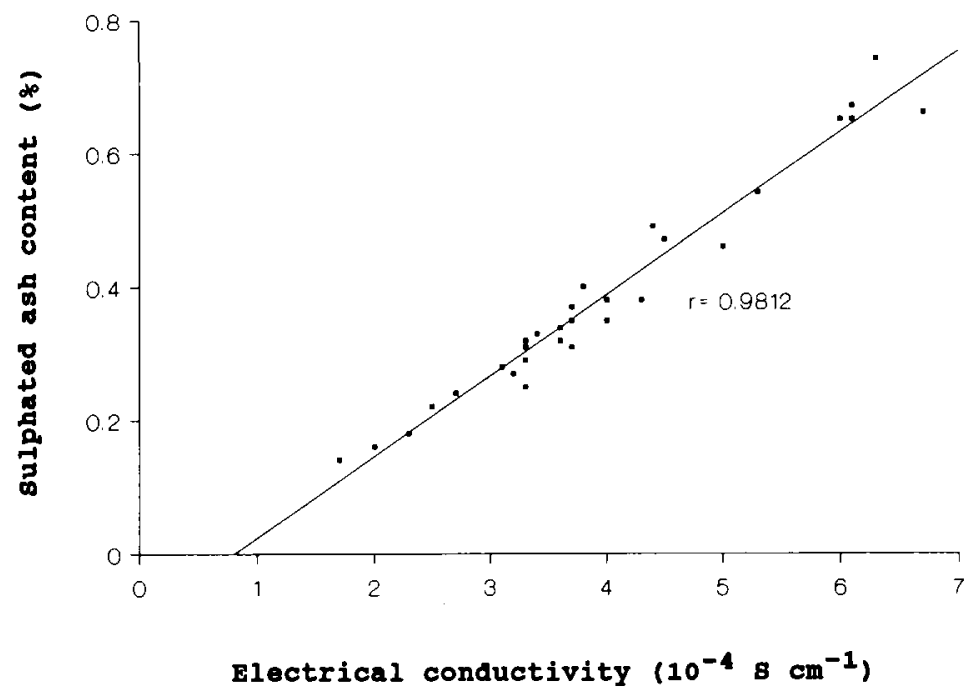

Fig 2. Graph of relation between electrical conductivities $\left(10^{-4} \mathrm{~S} \mathrm{~cm}^{-1}\right)$ of Basque honey solutions in humid matter and their sulphated ash contents (\%). 
from other locations and different harvests, and if the values of the coefficients change with time.

\section{ACKNOWLEDGMENTS}

We thank the Departamento de Agricultura $y$ Pesca of the Basque Government for the grant awarded to Maria Teresa Sancho Ortiz and the Associations of Beekeepers of the Basque country for providing the samples for the study.

Résumé - Relations entre la conductivité électrique et la teneur en cendre totale et sulfatée dans les miels basques. Dans le miel, la conductivité électrique est reliée au contenu en cendres, en acides organiques, en protéines, et en polyols (Crane, 1975). Accorti et al (1986, 1987) ont établi une relation entre la teneur en cendre totale et la conductivité électrique de solutions de miel à $20 \%$, exprimées à partir du poids sec du miel.

Dans la littérature, il n'y a aucune donnée concernant le contenu en sels minéraux des miels, exprimé en teneur en cendre sulfatée. La détermination de la teneur en cendre sulfatée (comme pour les sucres et les produits sucrés) conduirait à une perte moindre des composés volatils, et à une moindre incertitude dans les mesures, puisque la quantité absolue de cendres est plus grande.

La détermination de la teneur en cendre des miels est une opération longue et fastidieuse. Dans le travail présenté ici, on discute une possible relation entre la conductivité électrique, une mesure d'obtention rapide et aisée à $20^{\circ} \mathrm{C}$, et la teneur en cendre totale et sulfatée.

Cette étude concerne 30 miels basques récoltés à l'automne 1989, et originaires de la principale région de production. Ils ont été collectés, de manière représentati- ve, de régions de montagne, de régions côtières et de régions de plaine. L'analyse microscopique a révélé que 29 provenaient de nectars seuls, et 1 était un mélange de nectar et de miellat.

La conductivité électrique a été mesurée en solutions contenant $10 \mathrm{~g}$ de miel dissous dans $75 \mathrm{ml}$ d'eau bidistillée, puisque Sancho et al (1991) ont trouvé une excellente corrélation ( $r=0,9998)$ entre la conductivité électrique de ces solutions et celles mesurées dans des solutions de miel contenant $20 \%$ de matières sèches (Vorwohl 1964a et b, et Louveaux et al, 1973).

La teneur en cendre totale a été déterminée selon la méthode de White et al (1962), qui est la méthode officielle de ['AOAC pour le miel (1990).

La teneur en cendre sulfatée a été déterminée par la méthode de Leach et Winton (1920), qui est la méthode officielle de I'AOAC pour les sucres et les produits sucrés (1990).

La détermination des valeurs de la conductivité électrique à $20^{\circ} \mathrm{C}$ (tableau I), en poids frais, par rapport à la teneur en cendre totale (fig 1) et sulfatée (fig 2) des miels basques, donne une réaction linéaire dans les 2 cas.

$\%$ cendre totale $=0,083$ conductivité électrique $-0,092(r=0,964)$

$\%$ cendre sulfatée $=0,121$ conductivité électrique - 0,097 ( $r=0,981)$

Les valeurs théoriques des teneurs en cendre totale et sulfatée, calculées à partir de ces équations, sont également présentées dans le tableau I. Le test et» a été appliqué à ces données. Aucune différence significative (tableau II) n'a été montrée entre les 2 séries de résultats $(P=96,3 \%$ et $99,4 \%$, respectivement pour les cendres totale et sulfatée). Pour la teneur en cendre totale, Accorti et al (1986 et 1987) ont obtenu une relation similaire. 
Il a été montré que, au moins pour les miels basques récoltés à l'automne 1987 , la teneur des miels, en cendres totale et sulfatée, pouvait être estimée à partir de la valeur de la conductivité électrique à $20^{\circ} \mathrm{C}$, en matière fraiches.

Une extension de cette étude devrait être réalisée, afin de voir si la même relation existe pour les miels de différentes origines (nectar et miellat), de différentes provenances, et de différentes récoltes, et si les valeurs des coefficients changent avec le temps.

miel / conductivité électrique / teneur en cendre / Espagne

\section{Zusammenfassung - Beziehungen zwi-} schen elektrischer Leitfähigkeit und totalem wie sulfatiertem Aschengehalt in baskischen Honigen. Es besteht eine Beziehung zwischen elektrischer Leitfähigkeit (EI) und dem Gehalt an Asche, organischen Säuren, Protein und Polyol im Honig (Crane 1975). Accorti et al haben eine Beziehung zwischen dem totalen Aschengehalt und der El von $20 \%$ Honiglösungen, ausgedrückt im Trockengewicht des Honigs, festgestellt. In der Literatur gibt es keine Angaben über den Mineralgehalt von Honigen ausgedrückt als sulfatierte Asche. Die Bestimmung des Gehalts an sulfatierter Asche (zB für Zucker und Zuckerderivate) würde $z u$ einem geringeren Verlust an flüchtigen Komponenten führen (die in sulfatierter Form nicht sehr flüchtig sind) und zu geringeren Fehlern bei der Messung, da die absolute Aschenmenge größer ist. Die direkte Bestimmung des Aschengehalts des Honigs ist zeitraubend und kostspielig. In der vorliegenden Untersuchung wird eine mögliche Beziehung zwischen $\mathrm{El}$, die sich bei $20^{\circ} \mathrm{C}$ rasch und einfach bestimmen läßt, und dem totalen und sulfa- tierten Aschengehalt diskutiert. Die Untersuchung umfaßt 30 baskische Honige, geerntet im Herbst 1987 in den Haupttrachtgebieten. Sie wurden aus Gebieten repräsentativ für die Berg-, Küsten- und Flachlandregion gesammelt. Wie die mikroskopische Analyse zeigte, stammten 29 Honige aus einer Nektartracht und einer aus einer gemischten Tracht von Nektar und Honigtau. Die El wurde in Honiglösungen mit 10 g Honig in $75 \mathrm{ml}$ zweifach destilliertem Wasser gemessen, da Sancho et al (1991) eine ausgezeichnete Übereinstimmung $(r=$ 0,9998 ) zwischen der El dieser Lösungen und der El von Honigiösungen mit $20 \%$ Trockensubstanz (nach Vorwohl, 1964a, b; und Louveaux et al, 1973) gefunden hatten. Der totale Aschengehalt wurde nach der Methode von White et al, 1962 bestimmt, der offiziellen Methode für Honiguntersuchungen nach AOAC (920.181, 1990). Der Gehalt an sulfatierter Asche wurde nach der Methode Leach und Winton, 1920 , bestimmt, der offiziellen Methode für Zucker und Zuckerprodukte der AOAC (900.02, 1990). Trägt man die Werte der El bei $20^{\circ} \mathrm{C}$ im Flüssigzustand (Tabelle I) gegen den totalen (Abb 1) und Ten sulfatierten (Abb 2) Aschengehalt der baskischen Honige auf, so erhält man in beiden Fällen eine lineare Relation:

$\%$ totale Asche $=0,083 \mathrm{El}-0,092(r=$ 0,964 )

$\%$ sulfatierte Asche $=0,121 \mathrm{El}-0,097(r=$ 0.981 ). In Tabelle I sind auch die theoretischen Werte der totalen und der sulfatierten Aschengehalte dargestellt, die nach diesen Gleichungen berechnet wurden. Diese Werte wurden einem $t$-Test unterworfen (BMDP.PD3 1985). Zwischen den beiden Serien ergab sich kein signifikanter Unterschied (Tabelle II; $P=96,3 \%$ und $99,4 \%$ für den totalen und den sulfatierten Aschengehalt). Für den totalen Aschengehalt erzielten Accorti et al, (1986, 1987) 
ähnliche Beziehungen. Es wurde somit nachgewiesen, daß zumindest für die baskischen Honige der Ernte des Herbstes 1987 totale und sulfatierte Aschengehalte aus den Werten der elektrischen Leittähigkeit bei $20{ }^{\circ} \mathrm{C}$ im flüssigen Zustand geschätzt werden können. Als Fortsetzung dieser Arbeit könnte geprüft werden, ob dieselben Beziehungen auch für andere Honige verschiedene Herkunft (Nektar oder Honigtau), aus anderen Gegenden und verschiedenen Ernten gelten und ob sich die Werte der Koeffizienten mit der Zeit verändern.

\section{Honiguntersuchung / elektrische Leitfä- higkeit / totaler und sulfatierter Aschen- gehalt}

\section{REFERENCES}

Accorti M, Piazza MG, Persano Oddo L (1986) Conduttività elettrica e ceneri nei mieli. Apicolt Mod 77 (4), 165-167

Accorti M, Piazza MG, Persano Oddo L (1987) La conductividad eléctrica y el contenido en cenizas de la miel. Apiacta 22, 19-20

Association of Official Analytical Chemists (1990) Official Methods of Analysis. AOAC Arlington, VA, USA, 15 th edn

BMDP (1985) Biomedical Computers Program. University of California Press, Berkeley, CA
Codex Alimentarius Commission (1969) Recommended European Standard for Honey. Joint FAOMHO Food Standards Program, Rome CAC/RS-12-1969; reprinted in Bee World (1970) 51, 79-91

Crane E (1975) Honey: A Comprehensive Survey. Heinemann, London

Leach AE, Winton Al (1920) Food Inspection and Analysis. 4th edn, p 654

Louveaux J, Pourtallier MP, Vorwohl G (1973) Méthodes d'analyses des miels. Conductivité. Bull Apic 16, 7 pp

Pearson D (1962) The Chemical Analysis of Foods. JA Churchill Ltd, 5th edn

Sancho MT, Muniategui S, Huidobro JF, Simal J (1991) Correlation between the electrical conductivity of honey in humid matter and dry matter. Apidologie (in press)

Vorwohl G (1964a) Die Messung der elektrischen Leitfähigkeit des Honigs und die Verwendung der Meßwerte zur Sortendiagnose und zum Nachweis von Verfälschungen mit Zucherfütterungs-honig. $Z$ Bienenforsch 7 , 37-47

Vorwohl G (1964b) Die Beziehungen zwischen der elektrischen Leitfähigkeit der Honige und ihrer trachtmässigen Herkunft. Ann Abeille 7 (4), 301-309

White JW Jr (1962) Determination of acidity, nitrogen and ash in honey. $J$ Assoc Off Agric Chem 45, 548-551

White JW Jr (1978) Honey. Adv Food Res 24, 287-375

Zerban, Sattler (1930) Ind Eng Chem Ana/ 2, 32 